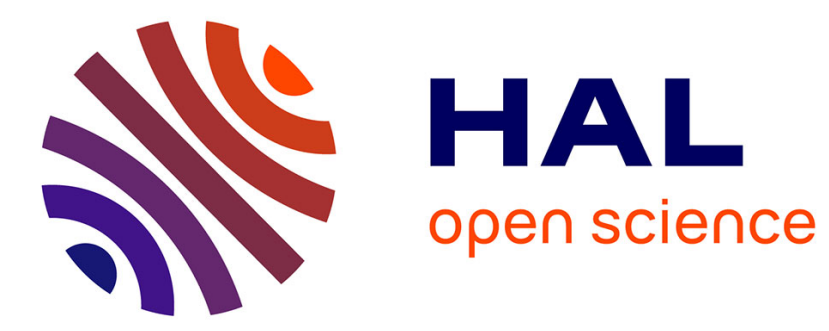

\title{
Multi-robots trajectory planning for farm field coverage
}

Christophe Cariou, Jean Laneurit, Jean-Christophe Roux, Roland Lenain

\section{To cite this version:}

Christophe Cariou, Jean Laneurit, Jean-Christophe Roux, Roland Lenain. Multi-robots trajectory planning for farm field coverage. 16th International conference on Control, Automation, Robotics and Vision (ICARCV), Dec 2020, Shenzhen, China. hal-03134018

\section{HAL Id: hal-03134018 \\ https://hal.inrae.fr/hal-03134018}

Submitted on 8 Feb 2021

HAL is a multi-disciplinary open access archive for the deposit and dissemination of scientific research documents, whether they are published or not. The documents may come from teaching and research institutions in France or abroad, or from public or private research centers.
L'archive ouverte pluridisciplinaire HAL, est destinée au dépôt et à la diffusion de documents scientifiques de niveau recherche, publiés ou non, émanant des établissements d'enseignement et de recherche français ou étrangers, des laboratoires publics ou privés. 


\title{
Multi-robots trajectory planning for farm field coverage
}

\author{
Christophe Cariou ${ }^{1}$, Jean Laneurit, Jean-Christophe Roux, Roland Lenain
}

\begin{abstract}
In the last few years, fleets of mobile robots have received increased interest in agriculture with the development of master/slaves control approaches. This paper proposes on the contrary a planning strategy enabling to generate beforehand the trajectory of each robot. For that, the fleet is considered as a single mobile entity with its steering and speed constraints. An admissible trajectory for this virtual entity, including maneuver phases, is generated to cover the shape of a given field. This one is next used to plan the trajectories of the actual robots. A panel of actual fields with different fleets of robots enables to highlight the relevance of the strategy proposed.
\end{abstract}

\section{INTRODUCTION}

Face to the need of sustainable development together with the constantly rising food demand of a steadily increasing world population (from 7.7 billion people in 2020 to 10 billion projected in 2050), world agricultural production is today confronted to numerous economic, ecological and societal challenges [1]. To fulfill some of these challenges, the potential of mobile robotics in terms of accuracy, repeatability and work capacity is increasingly put forward as a means to increase field efficiency and develop new environmentally friendly practices, as well as to relieve human operator from unhealthy operations [2], [3].

In the last few years, combined interventions of several agricultural robots in the same field have received increased interest to preserve soils from compaction and improve flexibility with the deployment of fleet of more or less numerous light robots [4], [5]. Immaterial towing of farm vehicles, as initially developed for convoy of trucks on highway [6] and usually called master/slaves convoy [7], is the main adopted strategy in the literature, see the illustration on Figure 1a: the master vehicle is manually driven by a human operator and the slave vehicles have to accurately follow its trajectory $\Gamma_{\text {master }}$ with respect to a desired curvilinear distance $s_{i}$, but also with respect to a lateral deviation $y_{i}$ depending on the working width of the vehicles, see [5], [8]. The main advantage of this approach is to not require a preliminary step of trajectory planning as the trajectory $\Gamma_{\text {master }}$ is created on-line, typically from the positions transmitted by radio link to the slave vehicles with a RTK GPS embedded on the master vehicle. The human driver of the master vehicle has however a major responsibility: if the master vehicle deviates, the followers will deviate too, leading to oscillations and inaccurate agricultural work. The human driver must also be aware of the kinematic and dynamic constraints of the following vehicles to generate a trajectory $\Gamma_{\text {master }}$ which will

\footnotetext{
${ }^{1}$ Authors are with University Clermont Auvergne, INRAE, UR TSCF, 9 av. Blaise Pascal CS 20085, F-63178 Aubiere, France. firstname. lastnamedinrae.fr
}

be feasible for the followers. In practice, such an approach involving shifted following vehicles is difficult to control for the human operator, especially during turns and maneuvers, but also in presence of obstacles and when the fleet is composed of a high number of vehicles. Moreover, farm fields can have complex irregular shapes leading to difficult on-line control of a fleet of robots in such environments [11].

The convoy on pre-assigned trajectories, illustrated on Figure $1 \mathrm{~b}$, is another strategy of multi-robots system: each vehicle has for mission to accurately follow its own previously planned trajectory $\Gamma_{A, B, C, \ldots, Z}$ and operates independently, eventually completed by a collision avoidance system or by imposing a safety inter-vehicle distance [9], [10]. The main advantage is that all the trajectories can be previously planned and adapted to both the field geometry, the known obstacles within the field, the steering and speed capacities of the vehicles as well as the agricultural tasks to be performed. The strategy can be to maintain a close formation by maintaining a desired distance between the vehicles, e.g. with the aim to facilitate the external monitoring, or not necessarily, e.g. to temporarily bypass an obstacle, maneuver or refill the tanks [10]. However, as the agricultural fields may have complex shapes, a preliminary analysis of the geometry of the field is a challenging part of this planning approach: in a single robot context, several work proposed to subdivide irregular fields into several elementary polygonal subregions wherein back and forth parallel straight paths are calculated with respect to the working width of the vehicle, and completed with different possibilities of travel directions and headland turning costs [11], [12]. A merge phase calculates next the possibility to aggregate or not the paths of adjacent subregions. The maneuvers are built considering the junctions of line segments and circular arcs which radius is constrained by the minimal turning radius of the vehicle. In addition, to plan fully feasible trajectories for a nonholonomic vehicle without curvature discontinuities, either spirals previously carried out by the actual vehicle or arcs of clothoid are inserted to take into account the motion constraints of the vehicle during turns [13], [14].

The convoy on pre-assigned trajectories involving several vehicles remains however poorly studied in the literature, most of the work focusing to adapt the methods developed in the automotive sector involving master/slaves convoys for the agricultural context. This paper proposes thus to address the issue of convoy on pre-assigned parallel trajectories considering homogeneous vehicles and aiming to cover polygonal shaped fields. To that end, a virtual robot is first defined, which steering and speed limitations gather those of the $n$ vehicles. Next, feasible continous curvature 


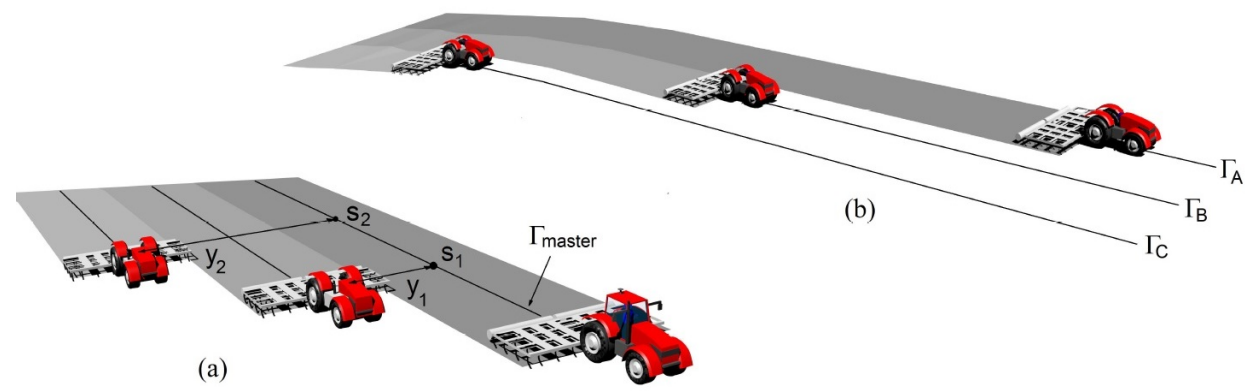

Fig. 1: Fleet of multi-robots in agriculture (a) Master-slaves convoy, (b) Convoy on pre-assigned trajectories

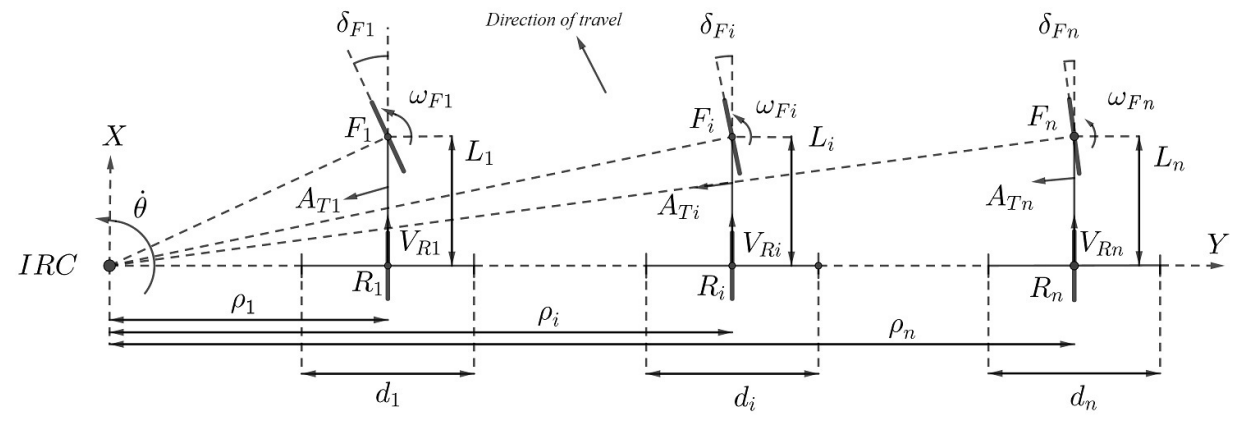

Fig. 2: Bicycle models of $\mathrm{n}$ vehicles positioned on the same IRC

trajectories based on adaptive clothoids are generated and the trajectories of U-turn for the fleet of vehicles are built. The main driving direction in the field is determined from a bruteforce algorithm. Finally, experimental fields with different shapes are processed to highlight the capabilities of the multirobots trajectory planner proposed.

\section{MODELING OF THE FLEET}

\section{A. Method}

To address the trajectory planning of multi-robots on preassigned parallel trajectories as depicted on Figure 1b, the method proposed in this paper is summarized in Figure 3. A virtual robot, positioned in the middle of the fleet and gathering the steering and speed constraints of the $n$ vehicles, is first considered. A trajectory generation algorithm suited for this virtual robot is then developed through the adaptation of the shape of clothoids with respect to the motion constraints of the robot. The U-turn maneuvers for the fleet in headland are next considered. A first strategy consists to maintain the geometrical layout of the fleet during the maneuver. A second one consists to realign the vehicles during the maneuvers, requiring to adapt the trajectories of each vehicle accordingly. In parallel, the analysis of the field shape enables to determine the driving direction of the fleet within the field from a brute force algorithm. The trajectories of each vehicle are finally generated.

\section{B. Modeling}

Let consider a fleet of $n \geq 2$ homogeneous car-like mobile robots, keeping a close formation as depicted on Figure 2. The vehicles, numbered from 1 to $n$, are represented

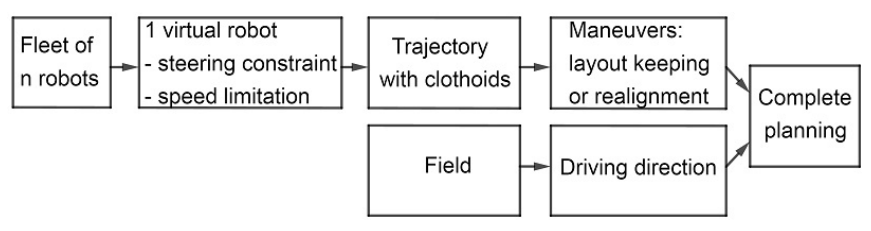

Fig. 3: Method followed

with their bicycle models (modeling assumption). They are positioned on the same instantaneous rotation center (IRC). The notations are as follows: for the vehicle numbered $i$, $R i$ and $F_{i}$ are respectively the centre of the rear and front axle. $L_{i}$ and $\delta_{F i}$ are respectively the wheelbase and the front wheel steering angle of the bicycle model, defining the instantaneous rotation center $(I R C)$ and the curvature radius $\rho_{i} . \delta_{\text {Fimax }}$ is the maximum reachable front steering angle, $\omega_{\text {Fimax }}$ is the maximum yaw rate of the front-wheel, $V_{R i}$ is the linear velocity at point $R_{i}$ and $A_{\text {Timax }}$ is the maximum transverse acceleration allowed during turns, see [14] for more details. $d_{i}$ is the working width of the vehicle.

\section{Motion constraints of the virtual robot}

Considering the vehicles side by side as depicted on Figure 2 , the distance $\Delta$ between the vehicles located at the extremities of the fleet is defined as follows:

$$
\Delta=\frac{d_{1}}{2}+d_{2}+\ldots+d_{i}+\ldots+d_{n-1}+\frac{d_{n}}{2}
$$

The virtual robot, which will be noted with the indice $\zeta$, is assumed to be located at the center of the fleet at $\Delta / 2$ (this position does not necessarily correspond to the position of a real robot with an even number of robot). 
1) Steering constraint: During a turn, the fleet is constrained by the maximum front steering angle of the vehicle located at the interior of the trajectory (by $\delta_{F 1 \max }$ on the configuration presented on Figure 2). In order that the steering angle requested for this interior vehicle not exceed this maximum value, a maximum steering constraint must be imposed on the virtual robot located at the center of the fleet. To define this value, let consider a fleet of homogeneous vehicles, i.e. same wheelbase $\left(L_{1}=L_{i}=L_{n}=\right.$ $L)$, same maximum reachable front steering angle $\left(\delta_{F 1 \max }=\right.$ $\delta_{\text {Fimax }}=\delta_{\text {Fnmax }}=\delta_{\text {Fmax }}$ ) and same maximum reachable speed $\left(V_{R 1 \max }=V_{\text {Rimax }}=V_{\text {Rnmax }}=V_{\text {Rmax }}\right)$. Let assume also that the wheelbase of the virtual robot is $L_{\zeta}=L$. It can be established:

$$
\tan \delta_{F \zeta}=\frac{L}{\rho_{\zeta}}=\frac{L}{\rho_{1}+\frac{\Delta}{2}}=\frac{L}{\frac{L}{\tan \delta_{F 1}}+\frac{\Delta}{2}}=\frac{1}{\frac{1}{\tan \delta_{F 1}}+\frac{\Delta}{2 L}}
$$

It comes the steering constraint for the virtual robot:

$$
\delta_{F \zeta \max }=\arctan \left(\frac{1}{\frac{1}{\tan \delta_{F \max }}+\frac{\Delta}{2 L}}\right)
$$

2) Speed constraint: The speed constraint of the virtual robot has next to be determined. In fact, during a turn, the fleet is constrained by the maximum speed of the vehicle located at the exterior of the trajectory. To not exceed this maximum value, let consider the heading variation $\dot{\theta}$ of the fleet for a left turn (i.e. $V_{R n}>V_{R 1}$ ):

$$
\dot{\theta}=\frac{V_{R n}-V_{R 1}}{\Delta}
$$

It comes:

$$
V_{R 1}=V_{R n}-\Delta \dot{\theta}
$$

Let consider now the velocity of the virtual robot:

$$
V_{R \zeta}=\frac{V_{R 1}+V_{R n}}{2}=V_{R n}-\frac{\Delta \dot{\theta}}{2}
$$

Moreover, the heading variation can also be considered for each vehicle, i.e.:

$$
\dot{\theta}=\frac{V_{R \zeta}}{\rho_{\zeta}}=\frac{V_{R 1}}{\rho_{1}}=\frac{V_{R n}}{\rho_{n}}
$$

Using (6), the equation (7) becomes:

$$
\begin{gathered}
V_{R \zeta}=V_{R n}-\frac{\Delta V_{R \zeta}}{2 \rho_{\zeta}} \\
V_{R \zeta}\left(1+\frac{\Delta}{2 \rho_{\zeta}}\right)=V_{R n} \\
V_{R \zeta}=\frac{V_{R n}}{1+\frac{\Delta}{2 L} \tan \delta_{F \zeta}}
\end{gathered}
$$

In the equation (10), $V_{R \zeta}$ depends on the velocity $V_{R n}$, i.e. the velocity of the vehicle located at the exterior of the turn, and on the steering angle $\delta_{F \zeta}$ of the virtual robot. The constraints on the maximum speed of the virtual robot will be defined considering the worst case, i.e. when the value of the steering angle $\delta_{F \zeta}$ is maximum. It comes:

$$
V_{R \zeta \max }=\frac{V_{R \max }}{1+\frac{\Delta}{2 L} \tan \delta_{F \zeta \max }}
$$

3) Note: The velocity of the vehicle located at the interior of the turn $V_{R 1}$ must moreover not be negative to avoid a reverse motion. The conditions (3) and (11) lead however that this situation never happens. In fact, let rewrite the equation (10) for the vehicle located at the interior of the turn:

$$
V_{R \zeta}=\frac{V_{R 1}}{1-\frac{\Delta}{2 L} \tan \delta_{F \zeta}}
$$

It comes:

$$
V_{R 1}=V_{R \zeta}\left(1-\frac{\Delta}{2 L} \tan \delta_{F \zeta}\right)
$$

$V_{R 1} \geqslant 0$ if $\left(1-\frac{\Delta}{2 L} \tan \delta_{F \zeta}\right) \geqslant 0$, leading to the condition:

$$
\tan \delta_{F \zeta} \leqslant \frac{2 L}{\Delta}
$$

The constraint on the steering angle of the virtual robot (3) can however be rewritten as follows:

$$
\tan \delta_{F \zeta \max }=\frac{2 L}{\Delta}\left(\frac{1}{\frac{2 L}{\Delta} \tan \delta_{F \max }+1}\right)
$$

$\left(\frac{2 L}{\Delta} \tan \delta_{F \max }+1\right) \geqslant 1 \forall \delta_{F \max } \in[0 \pi / 2$ [, i.e. the condition (14) is always satisfied.

4) Numerical example: To illustrate the constraints determined by the equations (3) and (11) on a fleet of robot, examples of numeric values are presented on Table 1, considering a fleet of $n \in[1 ; 10]$ homogeneous robots whose parameters are: $L=1.2 \mathrm{~m}, \delta_{F \max }=30^{\circ}, V_{R \max }=3.50 \mathrm{~m} . \mathrm{s}^{-1}$ and $d=$ $1 \mathrm{~m}$. Assuming for example a fleet of $n=4$ of such robots, the front steering angle of the virtual robot and its velocity must not exceed $18.5^{\circ}$ and $2.46 \mathrm{~m} . \mathrm{s}^{-1}$ respectively.

TABLE I: Steering and speed constraints on the virtual robot with respect to the number $n$ of robot in the fleet

\begin{tabular}{|c|c|c|c|}
\hline $\mathrm{n}$ & $\begin{array}{c}\Delta \\
(\mathrm{m})\end{array}$ & $\begin{array}{c}\delta_{F \zeta \max } \\
\left({ }^{\circ}\right)\end{array}$ & $\begin{array}{c}V_{R \zeta \max } \\
\left({\mathrm{m} . \mathrm{s}^{-1}}^{-1}\right.\end{array}$ \\
\hline 1 & 0 & 30.0 & 3.50 \\
2 & 1 & 24.9 & 2.93 \\
3 & 2 & 21.2 & 2.64 \\
4 & 3 & 18.5 & 2.46 \\
5 & 4 & 16.3 & 2.34 \\
6 & 5 & 14.6 & 2.26 \\
7 & 6 & 13.2 & 2.20 \\
8 & 7 & 12.1 & 2.15 \\
9 & 8 & 11.1 & 2.11 \\
10 & 9 & 10.3 & 2.07 \\
\hline
\end{tabular}

The generation of feasible trajectories for the virtual robot can thus be studied with the conditions (3) and (11). The parallel trajectories of each vehicle of the fleet will be then directly deduced from the trajectory of the virtual robot.

\section{Clothoids and maneuver strategies}

1) Clothoids: From the steering and speed constraints, feasible trajectories can be generated for the virtual robot. For that, the use of clothoid is particularly relevant as, on the one hand, the variation of the curvature $c$ of a clothoid is linear with respect to its curvilinear abscissa $s$ according to a constant of proportionality $g(c(s)=g . s)$ and, on the 


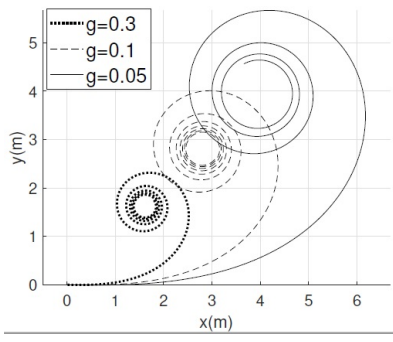

Fig. 4: Examples of clothoids. To calculate the cartesian coordinates of the curves, the Fresnel integrals are approximed from [15]

other hand, this curvature variation can be adapted with respect to the motion constraints of the vehicle, see (Levien, 2008), (Wilde, 2009), (Cariou, Gobor, Seiferth, and Berducat, 2017). Some examples of clothoids are presented on Figure 4 with different values for the constant of proportionality. In trajectory planning, arcs of clothoid enable to link elementary primitives as segment lines and circles. However, the value of the parameter $g$ has to be adapted to the steering and speed capabilities of the robot, as well as the curvature to be reached, e.g. to reach a circle of radius $1 / c_{o b j}$. The curvature $c_{o b j}$ can be determined for example with respect to the maximal steering angle of the virtual robot as follows:

$$
c_{o b j}=\frac{\tan \delta_{F \zeta \max }}{L}
$$

$g$ can be determined considering the time the robot will take to move on one centimeter at its maximal speed:

$$
t_{\text {timetodo } 1 \mathrm{~cm}}=\frac{0.01}{V_{R \zeta \max }}
$$

The maximum angle variation of the front wheel of the robot during this time is:

$$
\Omega_{\text {rotwheel }}=t_{\text {timetodol } \mathrm{cm}} * \omega_{F \zeta \max }
$$

That corresponds to the following curvature variation:

$$
d c=\frac{\tan \Omega_{\text {rotwheel }}}{L}
$$

Merging (17), (18), (19), and considering that $g=d c / d s$ with $d s=0.01 \mathrm{~m}$, it comes the value of $g$ to be chosen with respect to the motion capabilities of the robot:

$$
g=\frac{\tan \left(\frac{0.01 * \omega_{F \zeta \max }}{V_{R \zeta \max }}\right)}{0.01 * L}
$$

2) Different maneuver strategies: From (3), (11), (20), segment lines, arcs of clothoid and circles can thus be used to generate admissible trajectories for the virtual robot, respecting its motion constraints. Parallels to the trajectories thus generated will enable to deduce the admissible trajectories for the actual robots. This strategy can be used to create Uturn maneuvers conserving the layout of the fleet, see the examples on Figure 5. Loop and flat turns (Figures 5a and $5 b)$ enable adjacent back and forth trajectories in the field. Shifted turns, following the headland boundary, enable nonadjacent trajectories which can be calculated with respect to a multiple of the working width of the fleet, see Figure 5c.

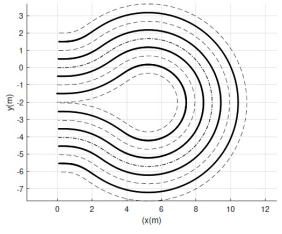

(a)

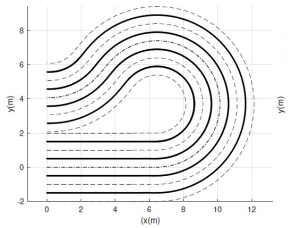

(b)

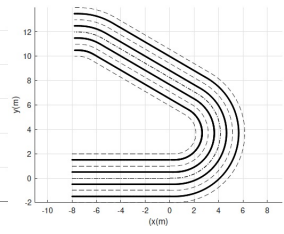

(c)
Fig. 5: Turn with $n=4$ vehicles maintaining their formation: (a) loop turn, (b) flat turn, (c) shifted turn following an headland angle of $30^{\circ}$. Plain lines: vehicles, dashed lines: working width, dasheddot lines at the center: virtual robot.

Another strategy consists to disrupt the layout of the formation to perform the U-turn maneuvers. The vehicles can for example be realigned during the maneuver to limit the required area width for turning, see Figure $6 \mathrm{a}$, or before the maneuver, see Figure $6 \mathrm{~b}$. In return, the high traffic involved in headland when the vehicles are realigned, all the more if a high number of vehicle is involved in the fleet, may increase the soil compaction phenomena in this area. This realignment strategy can however be used also to bypass obstacles or go through narrow passages (e.g. entrance of the field). Another strategy is to maneuver each vehicle independently, see Figure 6c.

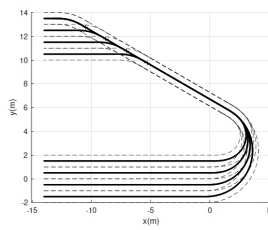

(a)

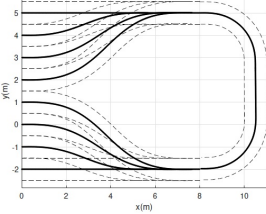

(b)

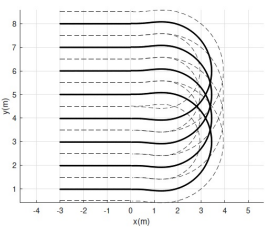

(c)
Fig. 6: Turn with $n=4$ vehicles, with realignment or in an independent manner: (a) realignment during the maneuver, (b) before the maneuver, (c) independently.

\section{E. Driving direction in the field}

Once the algorithm is operational to generate several shapes of admissible U-turn maneuvers for the fleet, the driving direction in the field has to be determined. For that, a brute force algorithm is used. It consists to rotate the original field from 1 to 360 degrees by step of 1 degree. At each step, the external rectangle parallel to the $\mathrm{x}$ and $\mathrm{y}$ axis including the field is determined and its area calculated. The kept rotation will be the rotation that satisfies at the three following conditions:

- The longest side of the rectangle is along the y-axis,

- The rotations leading to a discontinuity in the driving direction along the y axis are not kept,

- From the remaining rotations, the rectangle which has the minimal area is the soluction selected.

The driving direction of the fleet in the field is then defined as being along the $\mathrm{y}$ axis, and the $\mathrm{x}$ axis is sampled with respect to the working width, see the example on Figure 7. This approach is suited for fields having simple polygonal 
shapes (e.g. convex shapes) enabling to define a unique driving direction. No solution can however be found by the algorithm with field having complex concave shapes. That limitation will be studied in future work, requiring to determine more than a unique driving direction.

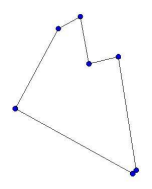

(a)

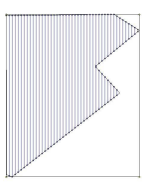

(b)

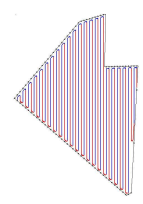

(c)
Fig. 7: Method to determine the driving direction of the fleet within the field: (a) original field, (b) solution not kept with discontinuity along the y-axis, (c) rotation selected and trajectory generated.

\section{RESULTS}

A panel of agricultural fields of our research institute have been considered to test the trajectory planning approaches presented in this paper, see Figures 8 and 9. The WGS84 coordinates of the field boundaries are first converted in metric ones using the Lambert conformal conic projection, which is a map projection for France. The coordinates of the vertices of the polygonal fields are given in Appendix A. The robots considered in the fleet are our experimental mobile robots presented on Figure 10. The main characteristics are a weight of $500 \mathrm{~kg}$, a wheelbase $L=1.2 \mathrm{~m}$, a maximum front wheel steering angle $\delta_{F \max }=30^{\circ}$, a maximum yaw rate of the front-wheel $\omega_{F \max }=45^{\circ} \mathrm{s}$ and a speed $V_{R \max }$ limited to $3.5 \mathrm{~m} . \mathrm{s}^{-1}$. The operating width is $d=1 \mathrm{~m}$.

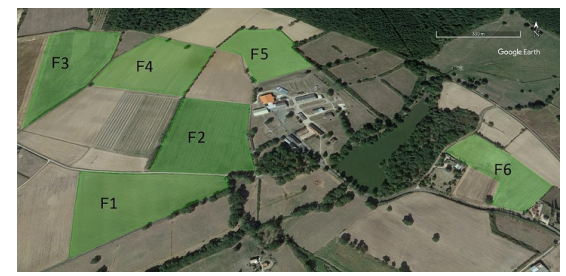

Fig. 8: Selected farm fields with different polygonal shapes Google Earth (c) Digital Globe, $46.336788^{\circ} \mathrm{N}, 3.430409^{\circ} \mathrm{E}$

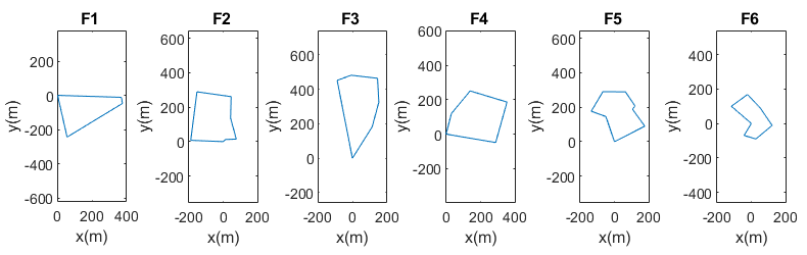

Fig. 9: Cartesian relative coordination systems

Figure 11 presents the results with $n=4$ robots processing the fields F1 and F2. To generate the trajectories, the maximum steering angle and speed limitations for the virtual robot were respectively $\delta_{F \zeta \max }=18^{\circ}$ and $V_{R \zeta \text { limit }}=2 \mathrm{~m} . \mathrm{s}^{-1}$ according to Table 1 . The chosen strategy was to maintain the layout of the fleet during the maneuvers. The algorithm determines automatically the driving direction in the field and generates the trajectories for each robot.

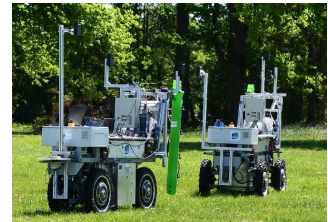

Fig. 10: Fleet of spraying mobile robots

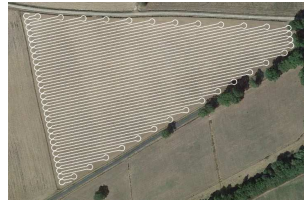

(a)

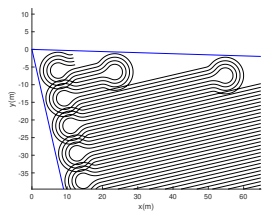

(c)

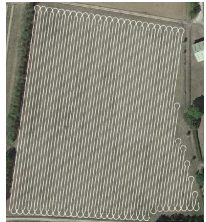

(b)

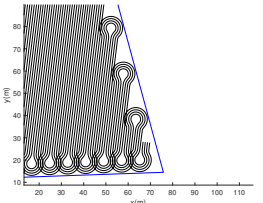

(d)
Fig. 11: Processing F1 and F2 with a fleet of $n=4$ robots. (a), (b): trajectory of the virtual robot, (c), (d): zoom on the trajectories generated for each robot.

Figure 12 considers $n=8$ robots and the fields F3 and F4. To generate the eight trajectories, the maximum steering angle and speed limitation for the virtual robot were defined respectively to $\delta_{F \zeta \max }=12^{\circ}$ and $V_{R \zeta \text { limit }}=2 \mathrm{~m} \cdot \mathrm{s}^{-1}$. The strategy was to maintain the layout of the fleet during the maneuvers. Figure 13 presents the results of $n=3$ robots processing the fields F5 and F6, with $\delta_{F \zeta \max }=21^{\circ}$ and $V_{R \zeta \text { limit }}=2 \mathrm{~m} . \mathrm{s}^{-1}$. The vehicles operate independently during the maneuvers.

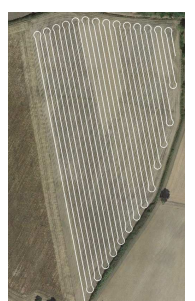

(a)

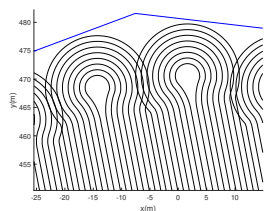

(c)

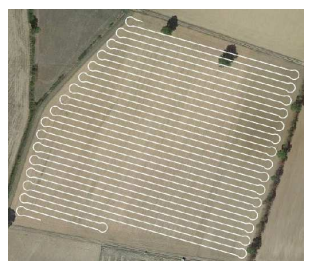

(b)

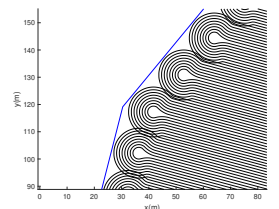

(d)
Fig. 12: Processing of the fields F3 and 4 with a fleet of $n=8$ robots. (a), (b): trajectory of the virtual robot, (c), (d): zoom on the trajectories generated for each robot. 


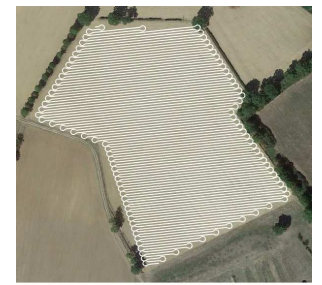

(a)

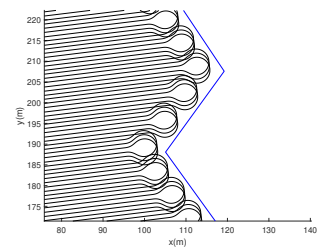

(c)

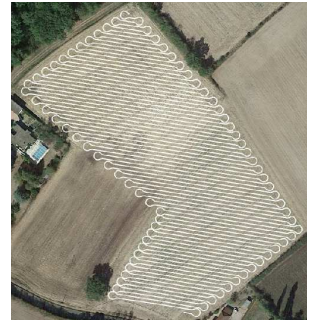

(b)

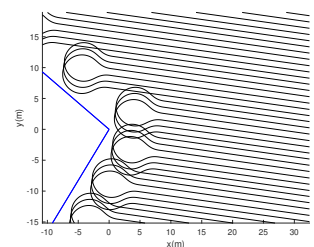

(d)
Fig. 13: Processing of the fields F5 and F6 with a fleet of $n=3$ robots, (a), (b): trajectory of the virtual robot, (c), (d): zoom on the trajectories generated for each robot.

\section{CONCLUSIONS}

The developement of fleet of autonomous light mobile robots is a promising way in agriculture to improve flexibility and preserve soil from compaction. The convoy on preassigned trajectories enables to adapt and anticipate the displacements w.r.t. both the field geometry, the steering and speed constraints of the robots and the agricultural task to be carried out. In that way, this paper considers $n \geq 2$ homogeneous vehicles and aiming to fully cover agricultural parcels. Based on the definition of a virtual robot, which steering and speed constraints gather those of the $n$ vehicles, continous curvature trajectories based on clothoids are generated and several layouts of maneuver are studied. The driving direction in the field is determined from a brute force algorithm, and admissible trajectories are finally built for each robot of the fleet. The approaches proposed are applied on several actual polygonal shaped fields, taking into account the steering and speed constraints of actual mobile robots. The main limitation of this work remains however the consideration of complex convex fields which requires to plan several driving directions of the robots in the field, or disrupt temporarily the fleet. Moreover, the number and type of robots to be engaged in a fleet could be optimized with respect to the geometry of the field and the working width. The trajectory planning of a fleet composed of nonhomogeneous mobile robots, with different working width and mobility capacities, could also be considered. Lastly, a collision avoidance system among the vehicles of the fleet needs also to be developed, in particular during U-turn maneuvers at the end of the fields, requiring to monitor the position and adapt the speed of each vehicle accordingly.

\section{ACKNOWLEDGEMENTS}

This work has been sponsored by the French government research program "Investissements d'Avenir" through the IDEX-ISITE initiative 16-IDEX-0001 (CAP 20-25), the IMobS3 Laboratory of Excellence (ANR-10-LABX-16-01) and the RobotEx Equipment of Excellence (ANR-10-EQPX44), and by the French National Research Agency under the grant number ANR-19-LCV2-0011 attributed to the joint Laboratory Tiara.

\begin{tabular}{|c|c|c|c|c|c|}
\hline \multicolumn{2}{|c|}{ Field F1 } & \multicolumn{2}{|c|}{ Field F2 } & \multicolumn{2}{|c|}{ Field F3 } \\
\hline $\mathrm{x}(\mathrm{m})$ & $\mathrm{y}(\mathrm{m})$ & $\mathrm{x}(\mathrm{m})$ & $\mathrm{y}(\mathrm{m})$ & $\mathrm{X}(\mathrm{m})$ & $\mathrm{y}(\mathrm{m})$ \\
\hline 0.00 & 0.00 & 0.00 & 0.00 & 0.00 & 0.00 \\
\hline 55.21 & -243.42 & 13.42 & 12.31 & 114.25 & 181.54 \\
\hline 377.79 & -47.33 & 75.83 & 14.49 & 153.22 & 324.77 \\
\hline \multirow[t]{4}{*}{371.91} & -11.45 & 42.75 & 136.49 & 145.49 & 463.81 \\
\hline & & 46.10 & 260.95 & -7.59 & 481.58 \\
\hline & & -152.02 & 288.94 & -88.44 & 451.44 \\
\hline & & -188.27 & 7.57 & & \\
\hline \multicolumn{2}{|c|}{ Field F4 } & \multicolumn{2}{|c|}{ Field F5 } & \multicolumn{2}{|c|}{ Field F6 } \\
\hline $\mathrm{x}(\mathrm{m})$ & $\mathrm{y}(\mathrm{m})$ & $\mathrm{x}(\mathrm{m})$ & $\mathrm{y}(\mathrm{m})$ & $\mathrm{X}(\mathrm{m})$ & $\mathrm{y}(\mathrm{m})$ \\
\hline 0.00 & 0.00 & 0.00 & 0.00 & 0.00 & 0.00 \\
\hline 287.37 & -48.84 & 175.76 & 90.14 & -40.96 & -67.74 \\
\hline 352.86 & 185.97 & 105.07 & 188.07 & -41.18 & -69.62 \\
\hline 139.12 & 250.76 & 119.22 & 207.59 & 26.29 & -89.86 \\
\hline \multirow[t]{4}{*}{30.43} & 119.15 & 63.57 & 289.57 & 120.64 & -11.48 \\
\hline & & -67.04 & 290.03 & 51.28 & 90.57 \\
\hline & & -134.38 & 176.89 & -22.49 & 168.24 \\
\hline & & -49.53 & 145.89 & -115.74 & 99.63 \\
\hline
\end{tabular}

Table 2: Vertices of the fields

\section{REFERENCES}

[1] FAO, The future of food and agriculture, trends and challenges. Food and Agriculture Organization of the United Nations, Rome, 2017.

[2] M. Bergerman, J. Billingsley, J. Reid and E.V. Henten. Robotics in Agriculture and Forestry. Springer handbook of rob., 1463-1492, 2016.

[3] T. Duckett, S. Pearson, S. Blackmore and B. Grieve. Agricultural robotics: the future of robotic agriculture. UK-RAS, 2018.

[4] M. Berducat, C. Debain, R. Lenain and C. Cariou. Evolution of agricultural machinery: the third way. 7th European Conference on Precision Agriculture, ECPA, pp. 363-369, 2009.

[5] A. Guillet, R. Lenain, B. Thuilot and V. Rousseau. Formation control of agricultural mobile robots: a bidirectional weighted constraints approach. Journal of Field Robotics, 7(34):1260-1274, 2017.

[6] N. Noguchi, J. Will, J. Reid and Q. Zhang. Development of a masterslave robot system for farm operations. Computers and Electronics in Agriculture, 44:1-19, 2014.

[7] M. Muratori, J. Holden, M. Lammert and A. Duran. Potentials for platooning in US. Highway freight transport. SAE Int. Journal of Commercial Vehicles, 10(1), 2017.

[8] X. Zhang, P. Noack and L. Grandl. A semi-autonomous tractor in an intelligent master-slave vehicle system. Intelligent Service Robotics, pp. 263-269, 2010.

[9] L. Emmi , M. Soto, G. Pajares and P. Santos. New trends in robotics for agriculture: integration and assessment of a real fleet of robots. The Scientific Worlds Journal, 2014.

[10] J. Conesa-Muñoz, G. Pajares and A. Ribeiro. Mix-opt: a new route operator for optimal coverage path planning for a fleet in an agricultural environment. Expert Systems With Applications, 50:364-378, 2016.

[11] T. Oksanen and A. Visala. Path planning algorithms for agricultural field machines. Helsinki University of Technology Automation Technology Laboratory, Research Report, 2007.

[12] J. Jin. Optimal field coverage path planning on 2D and 3D surfaces. Graduate Theses and Dissertations, 2009.

[13] J. Backman, T. Oksanen and A. Visala. Path generation method with steering rate constraint. In Proceedings of the International Conference on Precision Agriculture, Indianapolis, IN, 2012.

[14] C. Cariou, Z. Gobor, B. Seiferth and M. Berducat. Mobile robot trajectory planning under kinematic and dynamic constraints for partial and full field coverage. Journal of Field Robotics, 2017.

[15] K.D. Mielenz. Computation of Fresnel integrals. Journal or Research of the National Institute of Standards and Technology, 102(3):363-365, 1997. 